\title{
EFFECT OF HUMIDIFIER TEMPERATURE DURING NON-INVASIVE VENTILATION ON WATER CONDENSATE AND AIRWAY PRESSURE: NEWBORN MANIKIN MODEL
}

\author{
Teerawat Tangroekwarasakul, Thanin Pirunnet, Sangkae Chamnanvanakij \\ Department of Pediatrics, Phramongkutklao College of Medicine, Bangkok, Thailand
}

\begin{abstract}
Recommendation for setting humidifier temperature when using non-invasive ventilation in newborn infants has been changed from low range $\left(32-34^{\circ} \mathrm{C}\right)$ to high range $\left(37^{\circ} \mathrm{C}\right)$. However, there is no evidence comparing benefit and hazard between the two settings. To compare the effect of two temperature settings of humidifier during non-invasive ventilation on nasopharyngeal temperature, water condensate and airway pressure. We applied nasal intermittent positive pressure ventilation in a newborn manikin with artificial lungs. The temperature of humidifier was set at high range or low range. We measured nasopharyngeal temperature, water condensate in ventilator circuit and airway pressure in an artificial lung at 0,8 and 16 hours after starting ventilation. The experiments were conducted 3 times for each setting of humidifier. Comparisons between the groups were analyzed by using unpaired t-test or Mann Whitney test. Correlation of water condensate and airway pressure was analyzed by using Pearson's correlation. Nasopharyngeal temperature with humidifier temperature at high range $34.0 \pm 0.1^{\circ} \mathrm{C}$ was significantly higher than that at low range $32.1 \pm 0.2^{\circ} \mathrm{C}(p<0.001)$. Volume of water condensate in ventilator circuit was strongly correlated with airway pressure in artificial lungs ( $r=-0.828$ for PIP, $\mathrm{r}=-0.948$ for PEEP). Water condensate in ventilator circuit significantly decreased airway pressure and interfered with ventilator function. When using non-invasive ventilation, setting humidifier temperature at high range produces substantial water condensate resulting in significantly decreased airway pressure. Therefore, water drainage should be emphasized as animportant respiratory care. Funding: Phramongkutklao Foundation
\end{abstract}

Keywords : Humidifier, Non-invasive ventilation

J Southeast Asian Med Res 2017;1:20-24.

http://www.jseamed.org

\section{Correspondence to:}

Chamnanvanakij S, Department of Pediatrics, Phramongkutklao College of Medicine, Bangkok, Thailand E-mail : chamnanvanakij@gmail.com 


\section{Introduction}

When upper airway is bypassed during invasive ventilation or mechanical ventilation with endotracheal tube in place, warmed and humidified gas is necessary to prevent airway and lung injury. It is suggested that ventilatory devices should provide inspired gas to the lungs at temperature of $37^{\circ} \mathrm{C}$ and $100 \%$ relative humidity $(44 \mathrm{mg}$ $\left.\mathrm{H}_{2} \mathrm{O} / \mathrm{L}\right)^{(1-5)}$ This recommendation has been generally practiced in all age groups of patients including newborn infants. ${ }^{(6-8)}$ For patients requiring non-invasive ventilation (NIV) via facial mask, nasal prong ornasal cannula, there is no conclusive recommendation for setting optimal temperature and humidity of inspired gas. ${ }^{(4,5)}$ In adult, humidifier temperature is usually set at low range $(31-34 \mathrm{C})$ since the inspired gas will be warmed and humidified in the upper airway. Currently, a new guideline for newborn infants receiving NIV has been changed to set humidifier temperature similarly to invasive ventilation at $35.5-42 \mathrm{C}$ (average $37^{\circ} \mathrm{C}$ ). A proposed rationale is that newborn infants with rapid respiratory rate may have limited ability to warm inspired gas in their upper airway.

However, there was no study to determine benefit and harm of this guideline. High temperature of inspired gas may result in increased water condensate in ventilator circuit which subsequently increased risk of aspiration and infection. Since appropriate setting of humidifier temperature during NIV in newborn infants was controversial, we conducted an experiment to determine the effect of humidifier temperature on water condensate and airway pressure during NIV in a neonatal manikin model.

\section{Methods}

We conducted an experimental model by using a conventional ventilator (Evita V $300^{\circ}$, Dräger) with a humidifier (MR $850^{\star}$, Fisher-Paykel). The humidifier had 2 temperature setting modes: invasive and non-invasive modes Fig 1. A neonatal manikin with artificial lungs was placed in an infant incubator (Isolette $8000^{\circ}$, Dräger). Temperature and humidity at the nasopharyngeal level was measured by using a thermohygrometer (SK - L200THII $a^{18}$ humidity and temperature with SK - LTHII - 3 probe, SATO). Airway pressure in an artificial lung was measured by using a gas flow analyzer (FLUKEVT mobile gas flow analyzer $\left.^{8}\right)$ (Fig 2).
Volume of water condensate was measured directly after disconnecting ventilator circuit.

Nasal intermittent positive pressure ventilation (NIPPV) via nasal prong was set at peak inspiratory pressure (PIP) of 15 $\mathrm{mmHg}$, positive end expiratory pressure (PEEP) of $5 \mathrm{mmHg}$.

Room temperature and infant incubator were set at $26^{\circ} \mathrm{C}$ and $32^{\circ} \mathrm{C}$, respectively. The experiments were performed by setting humidifier temperature at either invasive or non- inva sive mode Fig 1.

Temperature and relative humidity at the nasopharyngeal level, volume of water condensate in ventilator circuit, airway pressure in an artificial lung and ventilatory pressure were sequentially measured at 8 and 16 hours after starting ventilation Table 1. The experiments were conducted 3 times for each setting of humidifier temperature.

Comparisons within each experiment were analyzed by using paired $t$-test or Wilcoxan sign rank test. Comparisons between the two settings of humidifier temperature were analyzed by using unpaired t-test or Mann Whitney test as appropriate. The association between volume of water condensate and airway pressure was analyzed by using Pearson's correlation.

\section{Results}

There were no differences in room temperature, relative humidity, and infant incubator temperature between the two groups of experiments. With humidifier setting at invasive mode, average temperatures of the inspiratory limb of venti- lator circuit were significantly higher than those with setting at non-invasive mode Table 2 .

With invasive mode, average temperature and relative humidity at nasopharyngeal level were significantly higher than those with non-invasive mode. Volume of water condensate in ventilator circuit was significantly higher with setting at invasive mode Table 3 .

Airway pressure, PIP and PEEP, in an artificial lung declined with time with setting at invasive mode whereas they were relatively constant with non-invasive mode. Declined PIP and PEEP returned to baseline levels after draining water condensate from both expiratory and inspiratory limbs of ventilator circuit Fig 3.

PIP and PEEP in an artificial lung were strongly correlated with volume of water condensate in ventilator circuit (correlation coefficient $=-0.828$ and -0.948 , respectively). 
Ventilatory pressures, PIP and mean pressure (Pmean), read on the monitor during invasive mode were significantly higher than those during non-invasive mode Fig 4 . In addition, waveform of ventilatory pressure on the monitor markedly fluctuated but returned to normal after draining water condensate from ventilator circuit.

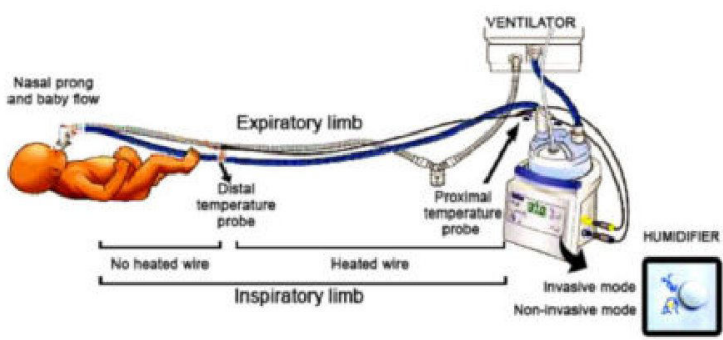

Fig 1 Experimental model: ventilator circuit (inspiratory and expiratory limbs), humidifier with two temperature settings (invasive and non-invasive modes), nasal prong and baby flow and a neonatal manikin

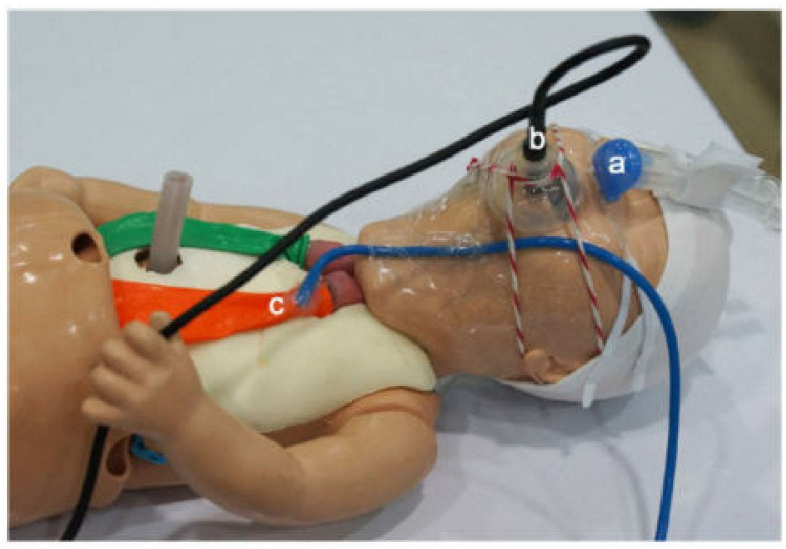

Fig 2 A neonatal manikin with nasal prong (a), thermohygrometer probe for measuring temperature and humidity at the nasopharyngeal level (b) and a gas flow analyzer probe for measuring airway pressure in an artificial lung (c)

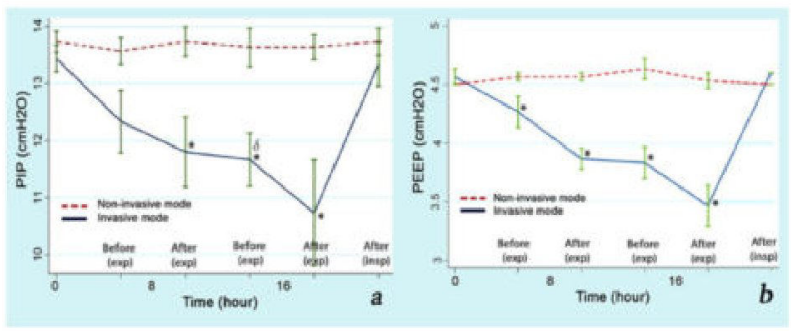

Fig 3 Comparisons of PIP (a) and PEEP (b) in an artificial lung between setting humidifier at invasive mode and non-invasive mode at 8 and 16 hours after starting ventilation (exp): draining water condensate in an expiratory water trap of ventilation

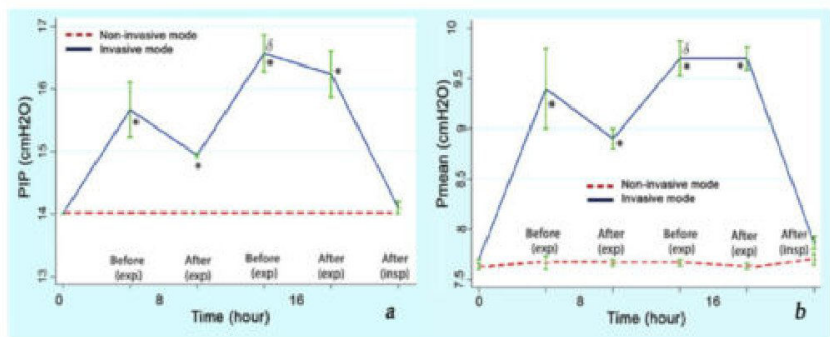

Fig 2 Comparisons of PIP (a) and mean presure (Pmean) (b) of the ventilator between setting humidifier at invasive mode and non-invasive mode at 8 and 16 hours after starting ventilation (exp): draining water condensate in an expiratory water trap o

Table 1 Timing and procedures to measure the outcomes of each experiment

$\begin{gathered}\text { Timing after starting } \\ \text { ventilation }\end{gathered}$
$\begin{gathered}\text { Temperatur } \\ \mathrm{e}\left({ }^{\circ} \mathrm{C}\right)\end{gathered}$

(exp): Draining water condensate in an expiratory water trap of ventilator circuit (insp): Draining water condensate in inspiratory limb of ventilator circuit

Table 2 Temperature and humidity of environment and inspiratory limb of circuit

\begin{tabular}{|c|c|c|c|}
\hline \multirow[t]{2}{*}{ Variables } & \multicolumn{2}{|c|}{ Humidifier temperature mode } & \multirow[t]{2}{*}{$p$-value } \\
\hline & Invasive & Non-invasive & \\
\hline \multicolumn{4}{|l|}{ Experimental environment } \\
\hline Room temperature $\left({ }^{\circ} \mathrm{C}\right)$ & $26.04 \pm 0.04$ & $25.98 \pm 0.06$ & 0.391 \\
\hline Room relative humidity (\%) & $75.29 \pm 0.56$ & $76.20 \pm 0.68$ & 0.337 \\
\hline Infant incubator temperature $\left({ }^{\circ} \mathrm{C}\right)$ & $32.00 \pm 0.00$ & $32.01 \pm 0.01$ & 0329 \\
\hline \multicolumn{4}{|l|}{ Temperature of inspiratory limb } \\
\hline Proximal probe $\left({ }^{\circ} \mathrm{C}\right)$ & $37.11 \pm 0.08$ & $31.58 \pm 0.25$ & $<0,001$ \\
\hline Distal probe $\left({ }^{\circ} \mathrm{C}\right)$ & $39.92 \pm 0.03$ & $33.92 \pm 0.04$ & $<0,001$ \\
\hline
\end{tabular}

Data presented as mean \pm standard error 
Table 3 Temperature and humidity at nasopharyngeal level and water condensate in circuit

\begin{tabular}{|c|c|c|c|}
\hline \multirow[t]{2}{*}{ Variables } & \multicolumn{2}{|c|}{ Humidifier temperature mode } & \multirow[t]{2}{*}{$P$ value } \\
\hline & Invasive & Non-invasive & \\
\hline \multicolumn{4}{|l|}{ Nasopharyngeal temperature $\left({ }^{\circ} \mathrm{C}\right.$ ) } \\
\hline 0 hour & $34.00 \pm 0.006$ & $32.07 \pm 0.19$ & $<0.001$ \\
\hline 16 hours & $34.50 \pm 0.06$ & $32.20 \pm 0.05$ & $<0.001$ \\
\hline \multicolumn{4}{|c|}{ Nasopharyngeal relative humidity (\%) } \\
\hline 0 hour & $99.00 \pm 0.00$ & $73.33 \pm 0.67$ & $<0.001$ \\
\hline 16 hours & $99.00 \pm 0.00$ & $73.00 \pm 0.29$ & $<0.001$ \\
\hline \multicolumn{4}{|c|}{ Volume of water condensate $(\mathrm{mL})$} \\
\hline 8 hour * & $33.23 \pm 1.16$ & $2.87 \pm 0.13$ & $<0.001$ \\
\hline 16 hours *" & $46.70 \pm 0.72$ & $2.53 \pm 0.15$ & $<0.001$ \\
\hline \multicolumn{4}{|c|}{ Data presented as mean \pm standard error } \\
\hline \multicolumn{4}{|c|}{ - Water condensate in an expiratory water trap of ventilator circuit } \\
\hline \multicolumn{4}{|c|}{ ** Water condensate in inspiratory and expiratory limbs of ventilator circuit } \\
\hline
\end{tabular}

\section{Discussion}

Currently, NIV including NIPPV, nasal continuous positive airway pressure (NCPAP) and nasal high flow was widely recommended for preterm infants requiring ventilatory support. In this study, we conducted an experiment by using NIPPV due to technical limitation in measuring airway pressure in a non-breathing manikin. This study demonstrated that humidifier setting at invasive mode generated gas temperature and humidity at the nasopharyngeal level close to recommendation. However, it is difficult to conclude that setting humidifier at invasive mode is better than non-invasive mode during NIV. In newborn infants with spontaneous breathing, temperature and humidity of inspired gas may increase in the nasal cavity and upper airway. ${ }^{(3,4)}$ With invasive mode, there was significant amount of water condensate in ventilator circuit including in the baby flow and nasal prong attached to the manikin. Water condensate decreased airway pressure in an artificial lung which was opposite to a previous study. Youngquist et al reported that water condensate in the expiratory limb of ventilator circuit increased airway pressure in the trachea. ${ }^{(9)}$ The contrary may be due to different ventilator circuit and methodology. The declined PIP and PEEP returned to baseline after draining water condensate from both the expiratory and inspiratory limbs of ventilator circuit. In addition, water condensate interfered with normal function of the mechanical ventilator.
The experiment was conducted in a manikin model under well-controlled environment. In a neonatal intensive care unit, lower room and incubator temperatures as well as exhaled gas may result in larger amount of water condensate and greater effect on the infant airway pressure. ${ }^{(10,1)}$

\section{Conclusion}

Our study demonstrated high humidifier temperature results in better airway temperature and humidity. However, it produces a large amount of water condensate which interrupts mechanical ventilator function and disturbs infant ventilation. Physicians should be aware of the benefit and harm of humidifier setting during receiving NIV. When setting humidifier temperature at high range, good respiratory care to minimize accumulation of water condensate in ventilator circuit should be emphasized.

\section{Acknowledgement}

This study was supported by Phramongkutklao College of Medicine. This study is registered at Thai Clinical Trials Registrywith ID: TCTR20160223001. We would like to thank the the Dräger Medical company for providing instruments and setting the experimental model.

Conflict of Interest

The authors declare no conflict of interest.

\section{References}

1. Gross JL, Park GR. Humidification of inspired gases during mechanical ventilation. Minerva Anestesiol 2012; 78: 496-502.

2. Branson RD, Gentile MA. Is humidification always necessary during noninvasive ventilation in the hospital. Respir Care 2010; 55: 209-16.

3. Williams R, Rankin N, Smith T, Galler D, Seakins P. Relationship between the humidity and temperature of inspired gas and the function of the airway mucosa. Crit Care Med 1996; 24: 1920-9.

4. American Association for Respiratory Care, Restrepo RD, Walsh BK. Humidification during invasive and noninvasive mechanical ventilation: 2012. Respir Care 2012; 57: 782-8.

5. Lellouche F, Maggiore SM, Deye N, Taillé S, Pigeot J, Harf A, et al. Effect of the humidification device on the work of breathing during noninvasive ventilation. Intensive Care Med 2002; 28: 1582-9. 
6. Davies MW, Dunster KR, Cartwright DW. Inspired gas temperature in ventilated neonates. Pediatr Pulmonol 2004; 38: 50-4.

7. Tarnow-Mordi WO, Reid E, Griftiths P, Wilkinson AR. Low inspired gas temperature and respiratory complications in very low birth weight infants. Pediatr 1989; 114: 438-42.

8. Jardine LA, Dunster KR, Davies MW. An experimental model for the measurement of inspired gas temperatures in ventilated neonates. Pediatr Pulmonol.2008; 43: 29-33.

9. Youngquist TM, Richardson CP, DiBlasi RM. Effects of condensate in the exhalation limb of neonatal circuits on airway pressure during bubble CPAP. Respir Care 2013; 58: 1840-6.

10. Todd DA, Boyd J, Lloyd J, John E. Inspired gas temperature during mechanical ventilation: effects of environmental temperature and airway temperature probe position. J Paediatr Child Health 2001; 37: 495-500.

11. O'Hagan M, Reid E, Tarnow-Mordi WO. Is neonatal inspired gas humidity accurately controlled by humidifier temperature? Crit Care Med 1991; 19: $1370-3$. 